\title{
Bus Dwell Time Estimation at Bus Bays: A Probabilistic Approach
}

\author{
Qiang Meng ${ }^{a l}$ and Xiaobo $Q u^{b}$ \\ ${ }^{a}$ Department of Civil and Environmental Engineering, National University of Singapore, \\ Singapore 117576 \\ ${ }^{b}$ Griffith School of Engineering, Gold Coast Campus, Griffith University, QLD 4222,
}

Australia

\begin{abstract}
The conventional data-driven regression approaches cannot be used to formulate the bus dwell time at a bus bay because they are incapable of dealing with the interactions among buses, arrival passengers, and traffic on the shoulder lane. Firstly, this paper points out that the bus dwell time at a bus bay possessed a high degree of uncertainty originating from the merging behaviour of bus to the vehicles in the shoulder lane. Secondly, it develops a novel probabilistic methodology to estimate the bus dwell time, including a standard regenerative stochastic process to model the interactions among buses, arrival passengers, and traffic on the shoulder lane. A tangible procedure is also proposed to estimate the mean and variable of the random bus dwell time. A case study is carried out to show the effectiveness of the proposed methodology. Finally, an impact analysis is carried out to demonstrate the significance of an advisory sign "give way to buses".
\end{abstract}

Key words: bus dwell time, regenerative process, gap acceptance theory, bus bays

\footnotetext{
${ }^{1}$ Corresponding author, Tel.: +65-6516 5494; fax +65 67791635

E-mail: ceemq@nus.edu.sg ( Qiang Meng); x.qu@griffith.edu.au (Xiaobo Qu).
} 


\section{Introduction}

The bus dwell time at a bus stop is defined as the time spent by a bus at the bus stop for passenger alighting and boarding, including time of opening and closing bus doors (Jaiswal et al., 2010). The bus dwell time is of great importance to estimate capacity of a bus station (Gu et al., 2011), and it is also a major component of bus travel time (Lin and Bertini, 2004; Hadas and Ceder, 2010; Tirachini and Hensher, 2011). In addition, the bus dwell time functions play a vital role in the transit assignment models (Lam and Bell, 2003; Kepaptsoglou and Karlaftis, 2009) and reliability analysis of the transit network (Szeto et al., 2011a\&b; Yan et al., 2013). Accordingly, the bus dwell time estimation is essential for bus operators and public transport planners (Ceder, 2007).

It seems that Levinson (1983) is the earliest study on the bus dwell time estimation. He formulated the bus dwell time as a function of two primary contribution factors - number of alighting and boarding passengers and the amount of time required for bus doors opening and closing - by using the linear regression approach. Since then, a number of case studies were carried out to take into account some secondary contributing factors for the bus dwell time estimation. For example, Guenthner and Hamat (1988) investigated the relationship between the bus dwell time and bus fare collection system. Levine and Torng (1994) analyzed impact of bus floor types on the bus dwell time. Jaiswal et al. (2010) examined influence of platform walking on bus rapid transit stations on bus dwell time. Tirachini (2011) studied impact of fare payment technology in urban bus services. It has been well recognized that the number of boarding or/and alighting passengers is the most significant contributing factors and the other parameters are the secondary contributing factors to the bus dwell time (Milkovits, 2008).

There is no doubt that the bus dwell time at a bus stop will be affected by layout of the bus top. According to TCRP Report 19 (1996), bus stops could be categorized into two types: 
curbside bus stops and bus bays. Curbside bus stops are the most common, simplest and convenient form of bus stops, which are located adjacent to the shoulder lane requiring only a sign to designate a stop. Due to its simple design, curbside stops are easy and inexpensive to install, easy to relocate, and provide easy access for bus drivers causing minimal delays to buses. However, they may potentially impede car flows and encourage drivers to make unsafe lane changing to avoid delay behind stopped buses. Bus bays are a specially constructed area separated from the traffic lanes and off the normal section of a roadway that provides for the loading and unloading of passengers (TCRP report 19, 1996). Bus bays allow the through traffic to move freely without obstruction of buses at bus bays and they should be provided primarily in high traffic volume or high-speed road ways such as urban expressways, or in heavily congested downtown where large number of passengers board and alight. Bus bays can be found everywhere in Singapore and they are also the prevailing public transport infrastructures in several major mega cities in Asia, including Hong Kong, Beijing and Tokyo because public transit is a major transport mode in these cities. The standard layout of a bus bay can be described by a trapezoid rotated by a 90-degree. As shown in Figure 1, a bus bay has three areas: bus entry area, alighting and boarding areas of passengers, and bus exit area. These entry and exit areas facilitate a bus to safely enter a bus bay from the shoulder lane and leave the bus bay to merge into traffic on the shoulder lane.

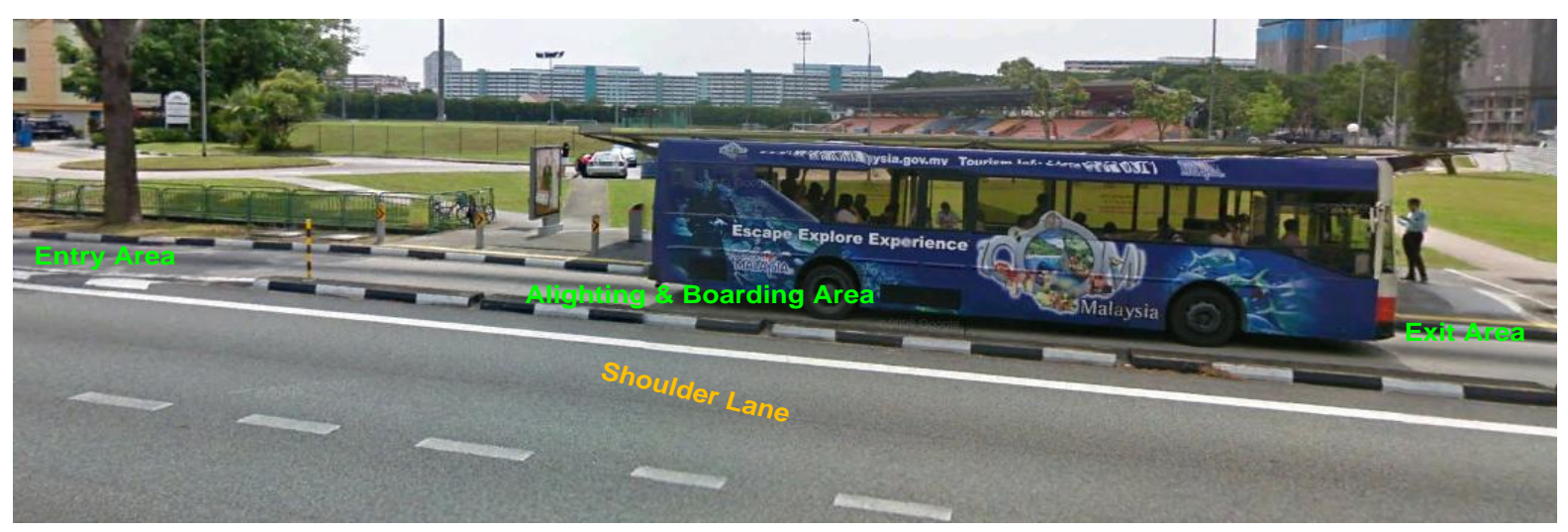

Figure 1. A bus bay picture taken in Singapore 
The bus dwell time at a bus bay is different from that at a curbside bus stop due to the unique interactions among the bus, arriving passengers, and traffic in the shoulder lane. After all the passengers alight and/or board the bus at a bus bay, the bus would leave the bus bay immediately to join traffic on the shoulder lane if possible. Different from buses in curbside bus stops, those in bus bays have to spend time on finding an acceptable time gap between consecutive vehicles on the shoulder lane. Meanwhile, it would be possible that a new passenger arrives at the bus bay when the bus is looking for an acceptable gap at the exit area. The bus driver has to re-open the entry door for this new passenger boarding in practical, suggested by the traffic act of Singapore ${ }^{2}$. After that, the bus would attempt again to leave the exit area and enter the shoulder lane. This bus entry door re-opening action can be often observed in Singapore due to its high population taking the public bus mode. It can be thus concluded that the bus bay should have a significant impact on the bus dwell time. In other words, it is of great significance for bus operators/transport authorities to estimate the bus dwell time at a bus bay.

In order to empirically investigate impact of bus bay on the bus dwell time, we have conducted a pilot survey study to collect the bus dwell time and number of boarding and alighting passengers associated with buses at one bus bay of Bus Line No. 188 at around 9:00 am on almost every working day from Mar $1^{\text {st }}$ to Jun $1^{\text {st }}, 2012$. We have collected 66 data in total because we only collect one data on every working day. These 66 data enable us to establish a linear relationship between the bus dwell time and number of alighting \& boarding passengers by the linear regression approach that has been widely used by the existing studies. Unfortunately, as can be seen in Figure 2, the linear relationship does not hold due to the relevant low coefficient of determination $\left(R^{2}=0.4875\right)$ and high root mean

\footnotetext{
${ }^{2}$ In reality, doors re-opening is what most drivers do practically according to our observation in many countries (Singapore, China, and Australia)
} 
square error $(\mathrm{RMSE}=3.48)$. Interestingly, these data scattered on a two-dimensional plane, in which $x$-axis denotes the number of boarding passenger and $y$-axis denotes the bus dwell time, apparently exhibits two regimes. The first regime is associated with the exactly one time for the bus entry door opening and closing. In the second regime, the bus entry door has been opened and closed for two times. Evidently, the bus dwell time at a bus bay may have more than one regime, which is caused by the number of times for the bus entry door opening and closing. With the same number of passengers, the bus dwell time in the Regime 1 is significantly less than that in the Regime 2 . In reality, the Regime 2 completely reflects the differences between the curbside bus stops and bus bays. It should be pointed out that $n$ regimes may be observable in principle provided that the entry door opens and closes for $n$ times.

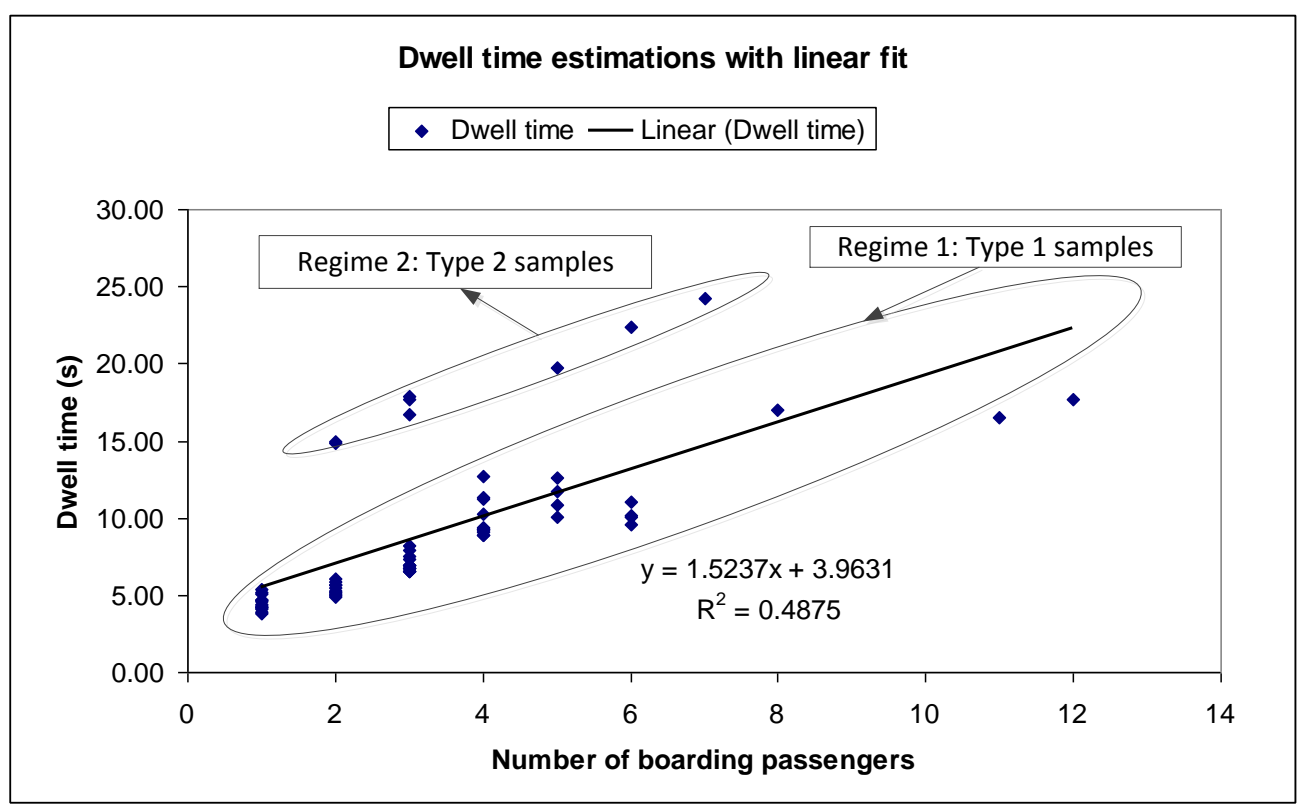

Figure 2. Dwell time vs. number of boarding \& alighting passengers

As analysed above, this pilot survey study fully shows that it is problematic to establish the relationship between dwell times and number of alighting and boarding passengers in bus bays using linear regression approaches. This is because the linear regression approaches are not able to take into account the interactions among buses, arriving passengers, and traffic on 
the shoulder lane. Therefore, it is of necessity to develop a new approach that can taking into account the interactions in bus dwell time estimation at a bus bay.

In this study, we propose a probabilistic approach to estimate dwell times of buses in a bus bay by incorporating the randomness caused by the interactions mentioned above. Different from the traditional dwell time estimation models, the dwell time will be represented by random variables. The contributions of this paper are twofold. Firstly, we propose a methodology to formulate the interactions among buses, arriving passengers, and the traffic in the shoulder lane. Secondly, the probability distribution types of bus dwell times are derived from the proposed regenerative stochastic process. The derived distributions of dwell time could be applied to estimate the bus stop capacity, analyse the travel time reliability of bus lines, and develop a stochastic transit assignment models.

\section{Interactions among Bus at a Bus Bay, Arrival Passengers and Shoulder Lane Traffic}

Let us use the bus bay shown in Figure 1 to elaborate interactions among three players: bus at the bus bay, passengers arriving at the bus bay and vehicles on the should lane. After entering the entry area, the bus will stop at the alighting \& boarding area and take the threestep operation: Step 1: open its doors; Step 2: passengers alight and/or board; and Step 3 close its doors. The bus will then move to the entry area and wait for occurrence of an acceptable gap to safety merge into traffic on the shoulder lane. An acceptable gap is defined as a time headway ( $T$ ) of two consecutive vehicles on the shoulder lane exceeding a critical $\operatorname{gap}\left(\tau^{*}\right)$, where $\tau^{*}$ is the least amount of time in which driver of the bus can comfortably and safely enters the shoulder lane. A new passenger may arrive at the bus bay during the time duration when the bus is waiting for an acceptable gap, and the bus is suggested to open the entry door to let the passenger board on the bus accordingly. Once the door re-opens, the subsequent process of boarding-waiting-entering will be irrelevant of the previous process. 
After that, the bus would attempt to enter the shoulder lane again. It could be possible that this process repeats again and again as shown in Figure 3. According to this figure, it can be seen that the process of the bus entering the shoulder lane is a typical regenerative stochastic process.

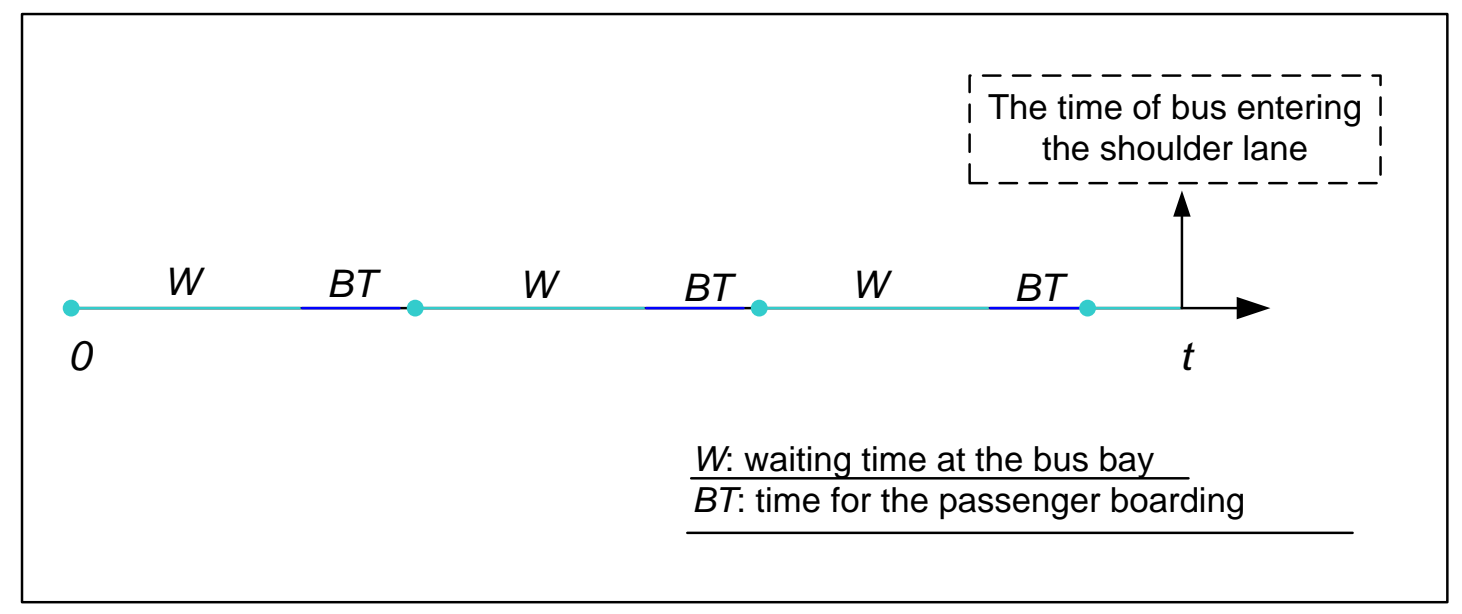

Figure 3. Process of bus entering the shoulder lane

It is practical and reasonable to assume that the time headway of vehicles on the shoulder lane $(T)$ and the inter-arrival time of passengers boarding on the bus at the bus bay $(Y)$ are two random variables following some distributions (e.g. exponential distribution). The bus driver's decision-making process regarding whether or not to enter the shoulder lane can be modelled by Bernoulli trials, namely, $K$, the number of unacceptable gaps before an acceptable one is observed by the bus driver, follows the geometric distribution with parameter $p$ :

$$
\operatorname{Pr}(K=k)=(1-p)^{k} p, k=0,1,2, \cdots, \infty
$$

where parameter $p$ is calculated by

$$
p=\operatorname{Pr}\left(T \geq \tau^{*}\right)
$$

Accordingly, the time spent by the bus at the exit area that is unable to merge into traffic on the shoulder lane is a random variable expressed by 


$$
W=\sum_{k=1}^{K}\left(T_{k} \mid T_{k}<\tau^{*}\right)
$$

Let $\rho$ denote the probability that a bus re-opens and re-closes entry doors at the bus bay when it waits for an acceptable time gap occurring on the shoulder lane at the exit area. This probability depends on the randomness of the inter-arrival time of passengers boarding on the bus at the bus bay. The probability $\rho$ is equal to the probability that a boarding passenger arrives at the bus bay during the time spent by the bus to merge into traffic on the shoulder lane, and it can thus be calculated by

$$
\rho=\operatorname{Pr}(Y \leq W)=\operatorname{Pr}\left(Y \leq \sum_{k=1}^{K}\left(T_{k} \mid T_{k}<\tau^{*}\right)\right)
$$

According to the total probability theorem, it follows that

$$
\rho=\sum_{k=0}^{\infty}\left((1-p)^{k} \times p \times \operatorname{Pr}\left(Y \leq k\left(T_{k} \mid T_{k}<\tau^{*}\right)\right)\right)
$$

Let $N$ denote the number of times of bus doors opening and closing when a bus in the alighting \& boarding area serving passengers and the exit area merging to traffic on the shoulder lane. It can be easily seen that $N$ follows a geometric distribution with parameter $\rho$ shown in Eq. (5), namely:

$$
\operatorname{Pr}(N=n)=\rho^{n-1}(1-\rho), n=0,1,2, \cdots, \infty
$$

The total time spent by the bus to merge into traffic on the shoulder lane depends on the number of the number of times of bus doors opening and closing, called as the merging time denoted by $\delta(N, W)$, and it can be estimated by

$$
\delta(N, W)=\sum_{j=1}^{N-1} W_{j}
$$

\section{Probabilistic Model Building}

\subsection{Methodology}


Let $x_{a}$ and $x_{b}$ denote the number of alighting and boarding passengers, respectively. Let $\alpha$ be the average time for one passenger boarding or alighting and $\beta$ be the average time used for opening and closing the doors. As boarding and alighting activities are simultaneously using different doors, the greater value of $x_{a}$ and $x_{b}$ will dominate the process. In other words, $\alpha \max \left(x_{a}, x_{b}\right)$ represents the time spent for boarding and alighting activities. By incorporating the two random terms $N$ and $\delta(N, W)$, the random bus dwell time at a bus bay can be estimated by

$$
D(x, N)=\alpha \max \left(x_{a}, x_{b}\right)+\beta N+\delta(N, W)
$$

The distributions of inter-arrival time of arriving passengers $(Y)$ and headway distributions of shoulder lane traffic $(T)$ are obtainable from field survey. Thus the mean and variance of $D(x, N)$ could be derived from either analytical or simulation approaches.

Without loss of generality, we assume the headways and passenger arrival time follow exponential distributions with parameters $\lambda$ and $\mu$, respectively. Thus, the probability that a passenger arrives at the bus bay during the merging time ( $\rho$ ) could be calculated by eq. (5). Since the two random variables $T$ and $Y$ are independent, we have,

$$
\begin{aligned}
\rho=\operatorname{Pr}(k(T \mid T<\tau) \geq Y)=\int_{0}^{\tau} \int_{0}^{k t}\left\{\frac{[\mu \exp (-\mu z)] \times[\lambda \exp (-\lambda t)]}{1-\exp (-\lambda \tau)}\right\} d z d t \\
=\frac{1}{1-\exp (-\lambda \tau)} \int_{0}^{\tau} \lambda \exp (-\lambda t) \times(1-\exp (-\mu k t) d t \\
=1-\frac{\lambda(1-\exp (-(\lambda+\mu k) \tau))}{(\lambda+\mu k) \times(1-\exp (-\lambda \tau))}
\end{aligned}
$$

where $k$ and $K$ are defined in eq. (1).

Substituting the simplified form of eq. (9) into eq. (5), it yields

$$
\rho=\sum_{k=0}^{\infty}\left\{(1-p)^{k} \times p \times\left(1-\frac{\lambda(1-\exp (-(\lambda+\mu k) \tau))}{(\lambda+\mu k) \times(1-\exp (-\lambda \tau))}\right)\right\}
$$


Thus, the times of doors opening and closing $(N)$ follows a geometric distribution with parameter $\rho$, as represented in eq. (6).

The merging time $\delta(N, W)$ could be represented by

$$
\delta(N, W)=(N-1) \times W=(N-1) \times \sum_{k=1}^{K}\left(T_{k} \mid T_{k}<\tau\right)
$$

where the distributions of $N, K$, and $T_{k}$ are all available. Accordingly, the mean of dwell time could be estimated by

$$
\begin{aligned}
E(D(x, N)) & =\alpha \max \left(x_{a}, x_{b}\right)+\beta E(N)+E(\delta(N, W)) \\
& =\alpha \max \left(x_{a}, x_{b}\right)+\beta / \rho+E(\delta(N, W))
\end{aligned}
$$

where $E(\delta(N, W))$ could be calculated by

$$
\begin{aligned}
E(\delta(N, W)) & =E((N-1)) \times E\left(\sum_{k=1}^{K}\left(T_{k} \mid T_{k}<\tau\right)\right) \\
& =E((N-1)) \times E(K) \times E\left(T_{k} \mid T_{k}<\tau\right) \\
& =\left(\frac{\rho}{1-\rho}\right) \times\left(\frac{1}{1-p}\right) \times E\left(T_{k} \mid T_{k}<\tau\right) \\
& =\frac{\rho(\lambda(1-\exp (-\lambda \tau))-\tau \exp (-\lambda \tau))}{(1-\rho)(1-p)(1-\exp (-\lambda \tau))}
\end{aligned}
$$

The variance of dwell time could be estimated by

$$
\begin{aligned}
\operatorname{Var}(D(x, N)) & =\beta^{2} \operatorname{Var}(N)+\operatorname{Var}(\delta(N, W)) \\
& =\beta^{2} \frac{1-\rho}{\rho^{2}}+\operatorname{Var}(\delta(N, W))
\end{aligned}
$$

where $\operatorname{Var}(\delta(N, W))$ can be numerically estimated through Monte Carlo Simulation.

\subsection{Model Calibration}

The survey data at the bus stop of dwell time and its corresponding number of passengers are the most important data required for calibrating the two coefficients: $\alpha$ - time for one passenger boarding or alighting, and $\beta$ - time spent on doors opening and closing. The critical gap $\tau$ should be calibrated on the basis of the accepted and rejected gaps by the buses 
at the bus bay. The distributions of headway of traffic on the shoulder lane $(T)$ should also be analysed to obtain the parameter $p$ of random variable $K$ according to eq. (1). Thus, the time spent by a bus at the exit area to merge into traffic if no passenger arrives $(W)$ is available by eq. (3). In addition, the distributions of passengers' arrival rate $(Y)$ is needed for estimating the probability that a boarding passenger arrives at the bus bay during the time spent by the bus to merge into traffic on the shoulder lane $(\rho)$ according to eq. (10). Thus, the distribution of the number of times of doors opening and closing $(N)$ is obtainable by eq. Error! Reference source not found.. The merging time $\delta(N)$ and the dwell time at a bus bay $D(x, N)$ could be derived by eqs. (11) and (8), respectively.

\section{A Case Study}

\subsection{Calibration of the coefficients}

This study takes a bus bay, AYE 431, for Bus Line No. 188 as the site of data collection, located at the Ayer Rajah Expressway of Singapore. Figure 5 depicts the layout of the bus bay, including the entry area, alighting \& boarding area, and exit area. We first collected the bus dwell time and number of boarding \& alighting passengers associated with buses deployed on this line at around 9:00 am every morning from Mar $1^{\text {st }}$ to Jun $1^{\text {st }} 2012$.

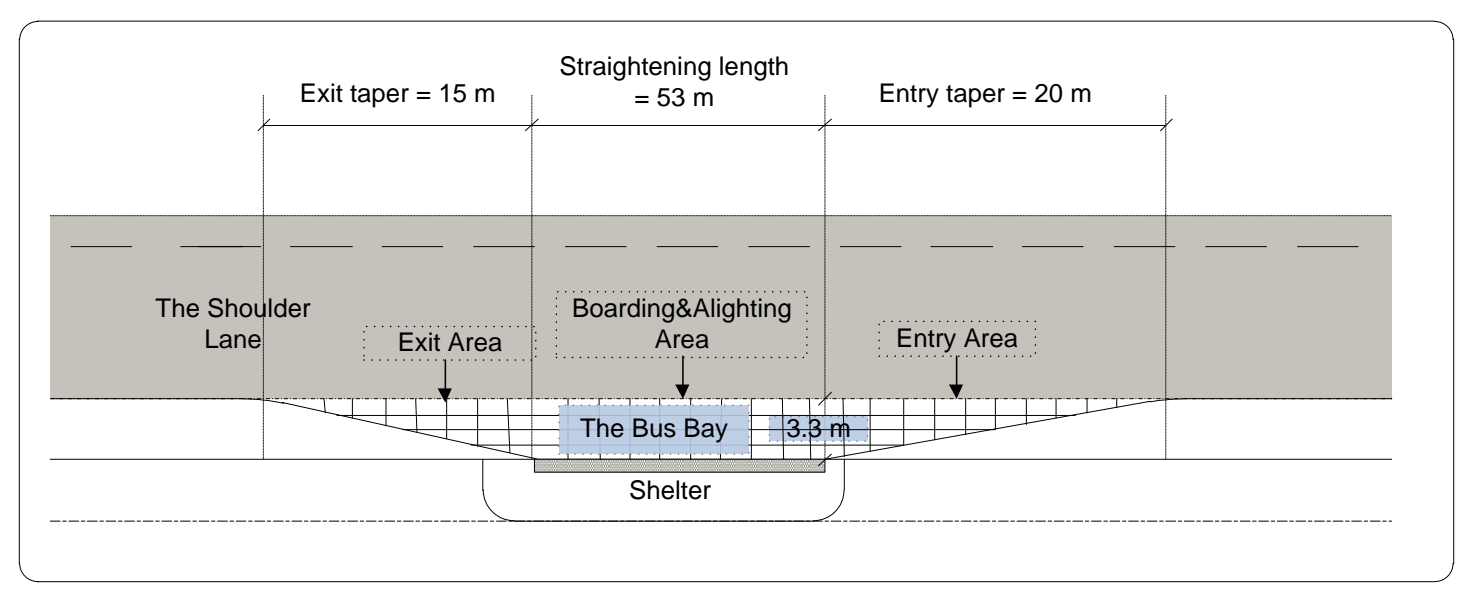


Figure 5. The bus bay station

As shown in Figure 6, buses serving this line have three doors - one entry door and two exit doors. According to the collected data, we find that the dwell time of a bus at the bus bay is determined by the number of boarding passengers. This is possibly caused by two reasons. Firstly, the boarding passengers are only allowed to get into the bus from the front entry door and alighting passengers usually get off the bus from the other two doors of a bus according to the Public Transit Regulations in Singapore. Secondly, the number of boarding passengers is generally more than that of alighting passengers at the bus bay during the survey time since the bus bay stations is nearby a residential area.

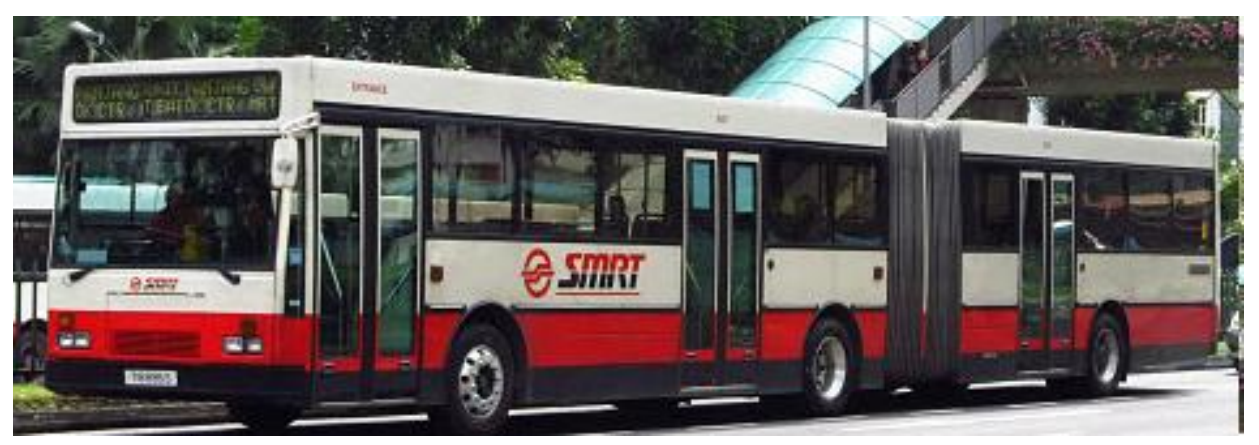

Figure 6. A bus deployed on Bus Line No. 188

Table 1 lists 66 data we have collected in the three months. Among the 66 data, there are 58 samples associated with exactly one time of doors opening and closing, called Type 1 data (the first 58 data), and 8 data samples related to two times for opening and closing the bus entry door, referred to as Type 2 data (the last eight samples).

Table 1. Dwell time data

\begin{tabular}{|c|c|c|c|c|c|}
\hline \# & $\begin{array}{l}\text { Number of } \\
\text { boarding passengers }\end{array}$ & $\begin{array}{l}\text { Dwell time } \\
\text { (s) }\end{array}$ & \# & $\begin{array}{l}\text { Number of } \\
\text { boarding passengers }\end{array}$ & $\begin{array}{l}\text { Dwell time } \\
\text { (s) }\end{array}$ \\
\hline 1 & 1 & 3.83 & 34 & 3 & 7.57 \\
\hline 2 & 1 & 3.89 & 35 & 3 & 7.88 \\
\hline
\end{tabular}




\begin{tabular}{|c|c|c|c|c|c|}
\hline 3 & 1 & 4.09 & 36 & 3 & 8.22 \\
\hline 4 & 1 & 4.18 & 37 & 4 & 12.75 \\
\hline 5 & 1 & 4.21 & 38 & 4 & 8.91 \\
\hline 6 & 1 & 4.3 & 39 & 4 & 8.94 \\
\hline 7 & 1 & 4.39 & 40 & 4 & 9.11 \\
\hline 8 & 1 & 4.58 & 41 & 4 & 9.14 \\
\hline 9 & 1 & 4.67 & 42 & 4 & 10.24 \\
\hline 10 & 1 & 4.7 & 43 & 4 & 11.25 \\
\hline 11 & 2 & 4.91 & 44 & 4 & 11.29 \\
\hline 12 & 2 & 4.98 & 45 & 4 & 9.31 \\
\hline 13 & 2 & 5.05 & 46 & 4 & 9.32 \\
\hline 14 & 1 & 5.1 & 47 & 4 & 9.37 \\
\hline 15 & 1 & 5.18 & 48 & 6 & 9.57 \\
\hline 16 & 2 & 5.22 & 49 & 5 & 10.02 \\
\hline 17 & 2 & 5.23 & 50 & 6 & 10.06 \\
\hline 18 & 1 & 5.34 & 51 & 6 & 10.17 \\
\hline 19 & 2 & 5.44 & 52 & 5 & 10.87 \\
\hline 20 & 2 & 5.68 & 53 & 6 & 11.05 \\
\hline 21 & 2 & 5.69 & 54 & 5 & 11.75 \\
\hline 22 & 2 & 5.87 & 55 & 5 & 12.62 \\
\hline 23 & 2 & 6.07 & 56 & 8 & 17.03 \\
\hline 24 & 3 & 6.56 & 57 & 11 & 16.47 \\
\hline 25 & 3 & 6.58 & 58 & 12 & 17.72 \\
\hline 26 & 3 & 6.59 & 59 & 2 & 14.85 \\
\hline 27 & 3 & 6.75 & 60 & 2 & 14.96 \\
\hline 28 & 3 & 6.76 & 61 & 3 & 15.40 \\
\hline 29 & 3 & 6.79 & 62 & 3 & 16.10 \\
\hline 30 & 3 & 6.9 & 63 & 3 & 16.70 \\
\hline 31 & 3 & 6.94 & 64 & 5 & 18.74 \\
\hline 32 & 3 & 6.98 & 65 & 6 & 18.34 \\
\hline 33 & 3 & 7.36 & 66 & 7 & 20.90 \\
\hline
\end{tabular}

The two coefficients, time for one passenger boarding $(a)$ and time for doors opening and closing $(b)$, can be calibrated by using the linear regression method. Figure 7 presents the results of the linear regression model. 


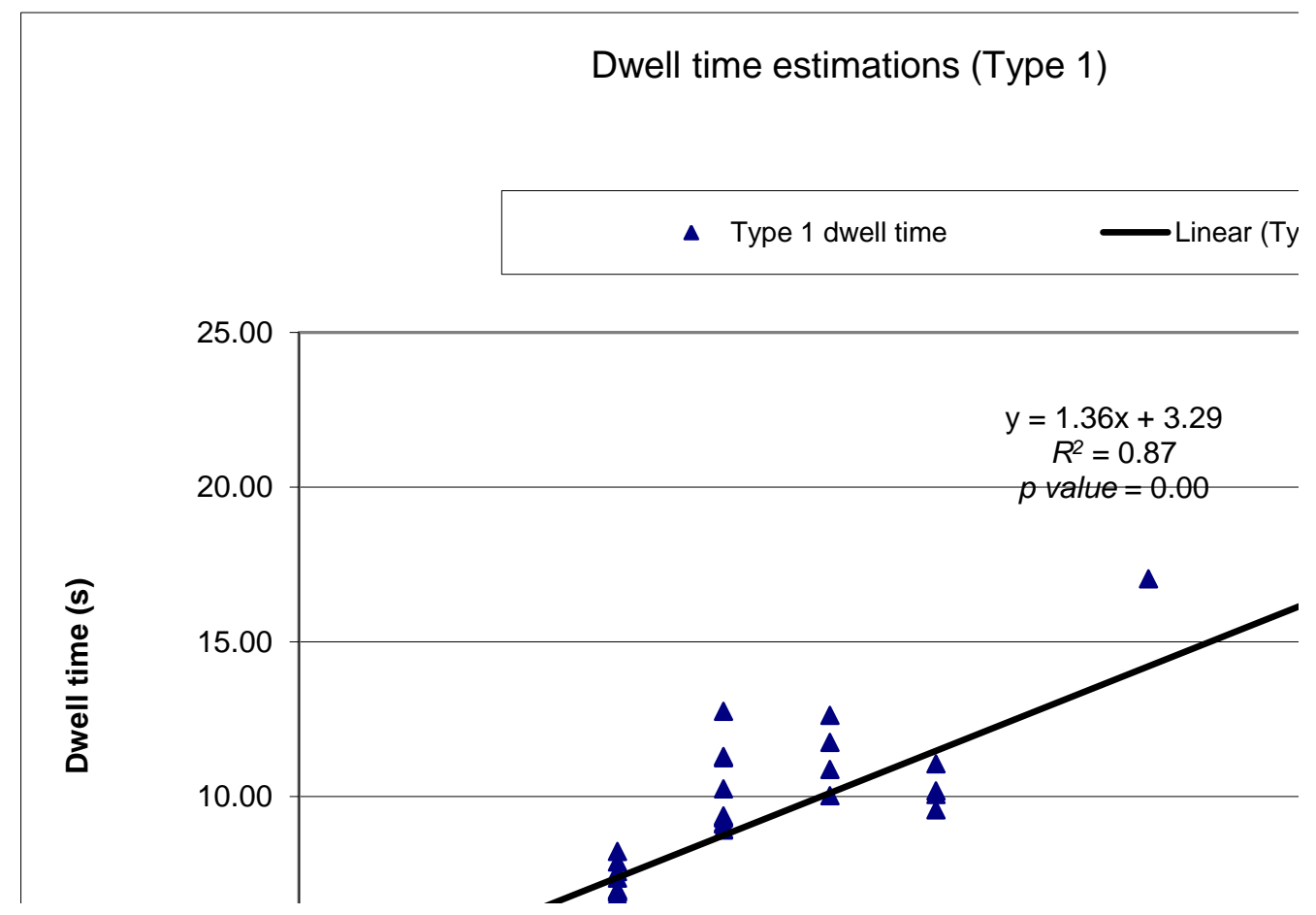

Figure 7. Dwell time functions for Type 1 data samples

It can be observed a fairly good linear trend for the Type 1 data samples $\left(R^{2}=0.87 ; p\right.$ value $=0.00$; and RMSE $=1.18$ ). As can be seen in the figure, the boarding time for one passenger $(a)$ is 1.36 and the time for doors opening and closing $(b)$ is 3.29 in this bus bay.

\subsection{Calculation of critical gap and the probability of bus entry door re-opening and re- closing}

In order to estimate the critical gap $\left(\tau^{*}\right)$ for this bus bay, the median critical gap (MCG) method (Lieu et al., 1999) is applied in this study. The method works as follows. Firstly, we draw the cumulative frequency that those accepted time headways are longer than a particular time threshold $\tau$, denoted by $F_{a}(\tau)$, for the accepted time headways (gaps). Then, the cumulative frequency that those rejected time headways are shorter than the time threshold $\tau$, 
denoted by $F_{r}(\tau)$, is also computed and depicted for the rejected gaps. The critical time gap $\tau^{*}$ should fulfil the equation below:

$$
F_{a}\left(\tau^{*}\right)=F_{r}\left(\tau^{*}\right)
$$

According to Figure 8, the critical time gap, i.e., solution of eq. (15), is around 5.8 seconds. We thus use 5.8 seconds as the critical gap in this case study.

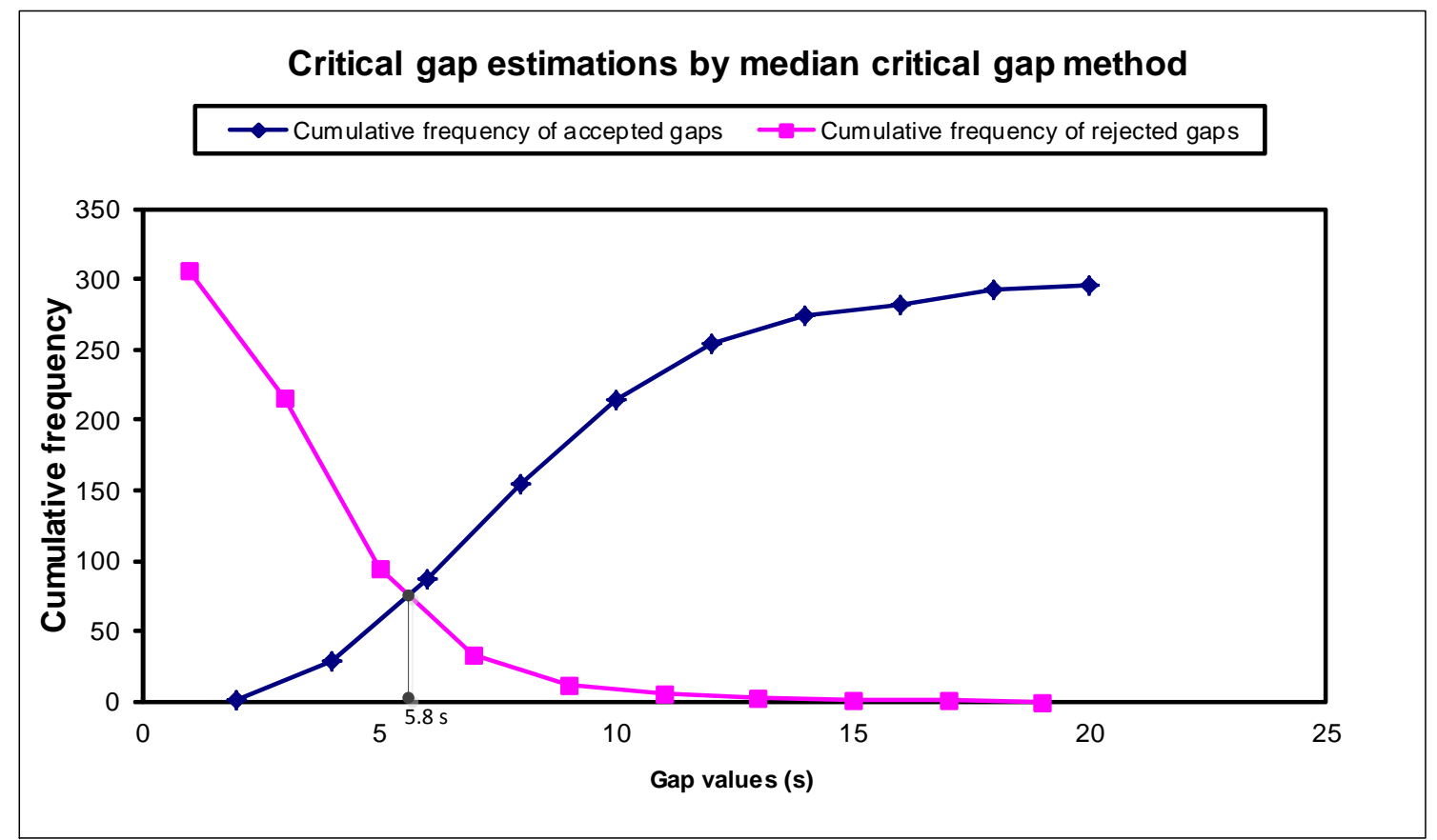

Figure 8. Critical gap estimation by the MCG method

The traffic volume on the shoulder lane during the survey time is around 540 vehicles/hour. As there is no traffic signal control in the AYE, it is reasonable to assume that the time headway of vehicles on the shoulder lane, denoted by $T$, follows an exponential distribution with mean of $3600 / 540=6.67$ seconds $^{3}$. According to eq. (2), the parameter $p$ can be estimated as

$$
p=\operatorname{Pr}(T \geq \tau)=\exp (-5.8 / 6.67)=0.42
$$

\footnotetext{
${ }^{3}$ Please refer to Lieu et al. (1999): traffic flow theory.
} 
Thus, the $K$ follows geometric distribution with the parameter $p=0.42$.

As introduced above, the passengers may arrive during the merging time of buses from exit area to the traffic in the shoulder lane. The field survey data suggest that the arrival rate of passengers, $Y$, follows an exponential distribution with mean of 36.0 seconds . Therefore, the probability that a passenger arrives at the bus bay during the merging time $(\rho)$ could be calculated by eq. (10).

$$
\rho=8.06 \%
$$

Thus, the times of doors opening and closing $(N)$ follows a geometric distribution with parameter $\rho=0.0806$.

\subsection{Probabilistic dwell time estimation}

In this case study, the dwell time in a bus bay $(D(x, N))$ is a random variable which is dependent on not only the number of boarding passengers but also the random variable $N$. Without loss of generality ( $x$ can take any values), we take $D(2, N)$ as an example to illustrate the randomness of dwell time. For this simplified case, there are only two boarding passengers $(\mathrm{x}=2)$. Accordingly, the times of doors opening and closing would not be

possible to exceed 2. That is to say, the sample space of random variable $N$ is $\{1,2\}$. According to eq. (10), the probability of exactly one time of doors opening and closing $(\operatorname{Pr}(N=1))$ is

$$
\operatorname{Pr}(N=1)=\rho^{(1-1)}(1-\rho)=1-\rho=0.9194
$$

Similarly, the probability of exactly two times of doors opening and closing $(\operatorname{Pr}(N=2))$ is 0.0806. The corresponding dwell times could be estimated by eq. (8).

$$
D(2,1)=1.3646 \times 2+1 \times 3.2899=6.02 \mathrm{~s}
$$




$$
D(2,2)=1.365 \times 2+2 \times 3.290+W=9.31 \mathrm{~s}+W
$$

where $D(2,2)$ is also a random variable. According to eqs. (13) and (14), the mean and variance of $D(2,2)$ could be estimated as follows.

$$
\begin{gathered}
E(D(2,2))=13.66 \text { seconds } \\
\operatorname{Var}(D(2,2)) \approx 36.52
\end{gathered}
$$

In sum, the expected value of dwell time $E(D(2, N))$ could be approximated by a discrete random variable, represented in Table 2.

Table 2. Random variable $E(D(2, N))$

\begin{tabular}{lll}
\hline$n$ & 1 & 2 \\
\hline$E(D(2, n))$ & 6.02 & 13.66 \\
$\operatorname{Var}(D(2, n))$ & 0 & 36.52 \\
Probability & 0.919 & 0.081 \\
\hline
\end{tabular}

To generalize, for any number of boarding passengers $x$ and any times of doors opening and closing $n$, the expected value of the dwell time could be estimated by

$$
\begin{aligned}
E(D(x, n)) & =1.36 \times x+n \times 3.29+(n-1) \times E(W) \\
& =1.36 \times x+n \times 3.29+(n-1) \times 4.36
\end{aligned}
$$

The variance with respect to $x$ passengers could be estimated numerically. The probability of $n$ times of doors opening and closing can be calculated by

$$
P(N=n)= \begin{cases}\rho^{n-1}(1-\rho), & \text { if } n<x \\ \rho^{n-1}, & \text { if } n=x\end{cases}
$$

The expected value of the bus dwell time at a bus bay is a discrete random variable due to the interactions among the buses, arriving passengers, and the traffic on the shoulder lane. 
The interactions can be formulated by a standard regenerative stochastic process and the distribution of the random variable could be derived. The variance and expected values of the dwell time can be estimated accordingly.

\subsection{Model verification}

According to Eqs. (23) and (24), the dwell time for this case study is derived, represented in Table 3.

Table 3 random variable $E(D(x, N))$

\begin{tabular}{lll}
\hline$E(D(x, n))$ & $E(D(x, 1))=1.36 \times x+3.29$ & $E(D(x, 2))=1.36 \times x+10.94$ \\
& & \\
\hline Probability & 0.9194 & 0.0806 \\
& & \\
\hline
\end{tabular}

According to the collected data, the percentage of Type 1 data samples is $87.9 \%$, which is around $4 \%$ lower than the calculated probability (0.9194). As can be seen in Figure 7, the $E(D(x, 1))$ is a good predictor for the dwell time for Type 1 data samples. We further conducted a comparative study to illustrate the performance of equation to predict the dwell time of Type 2 data samples $(E(D(x, 2)))$. As is shown in Figure 9, the model performs very well for the 8 Type 2 data samples $\left(\mathrm{R}^{2}=0.98 ;\right.$-value $=0.00$; and $\left.\mathrm{RMSE}=0.53\right)$. 


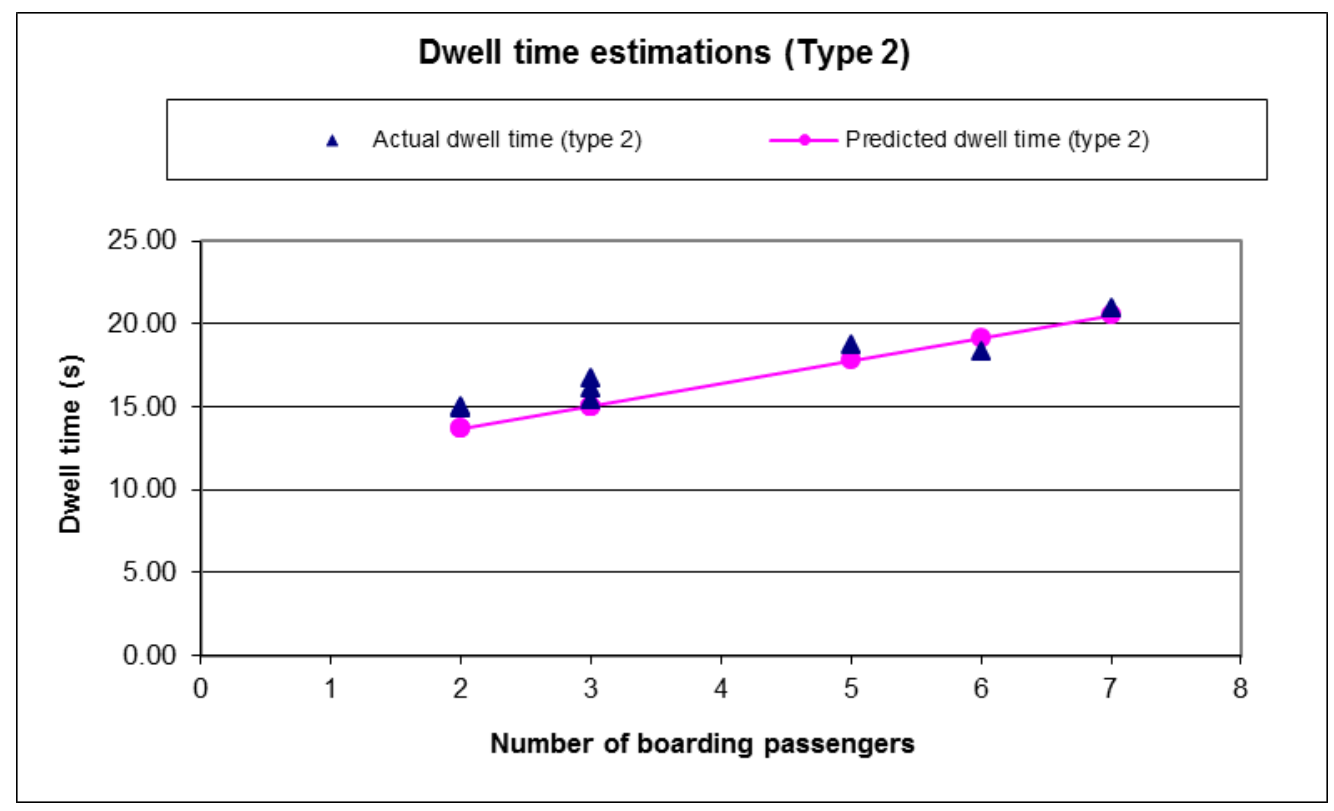

Figure 9: dwell time estimations (Type 2)

\section{Discussions and Implications}

\subsection{Impact analysis of higher traffic volume}

Theoretically, the probability of the occurrence of 3 or more times of doors opening and closing is very small according to eq. (24). In practice, this probability is probably even lower. This is because, in order to catch up with the schedule, the bus driver may not want to open the doors for three or more times even if new passengers arrive during the merging time. In reality, there is no such case out of all collected samples (66 samples). However, the multiple times of doors re-opening/re-closing might occur for more congested traffic conditions (e.g. in Beijing or Hong Kong). According to the eq. (9), the probability will be 0.30 if the travel volume in the shoulder lane and the passengers' arrival rate both double. Indeed, the Type 2 scenarios might frequently occur under high traffic volume if the vehicles in the shoulder lane do not give way to the buses.

Although bus bays facilitate the traffic flows in traffic lanes, they will significantly increase the bus dwell time. Accordingly, buses may not be able to enter into the traffic lanes before a new passenger arrives. This will result in the inapplicability of the traditional bus 
dwell time estimation models in the bus bays. According to the analysis, the time needed for drivers to enter into the shoulder lane follows a combined geometric-exponential distribution for bus bays in expressways of Singapore. The parameter of the distribution can be calibrated by gap acceptance theory. In addition, the interactions between buses and arriving passengers could be formulated by a standard regenerative stochastic process. As long as a new passenger arrives during the merging time, the systems will repeat the same process consisting of doors opening, passengers boarding, doors closing, and bus merging into the shoulder lane.

In practice, bus bays are very common in urban cities around the world. The methodology can be easily generalized and applied to estimate the dwell time for bus bay stations in other countries. However, the parameters (the critical gap value, the arrival pattern of customers, the dwell time samples, the number of boarding and alighting passengers, and the headway distributions of the traffic on the shoulder lane) need to be collected and estimated on the basis of the field survey. In addition, the time for each passenger boarding and the time for opening and closing doors should be calibrated by regression models.

\subsection{Implications - the impact of "give way to bus" advisory sign}

Under very congested scenarios, the dwell time might be very long due to the difficulty of finding an acceptable gap, thus not only causing significant delay but also resulting in the issues of bus stop capacity (the bus bay is occupied and arriving buses could not enter into the bay). This may lead to more serious problems for the traffic on shoulder lane. If an advisory sign "give way to bus" is posted at the bus rear (e.g. Figure 10), it will significantly change the mechanism among buses, arriving passengers, and traffic in the shoulder lane. 


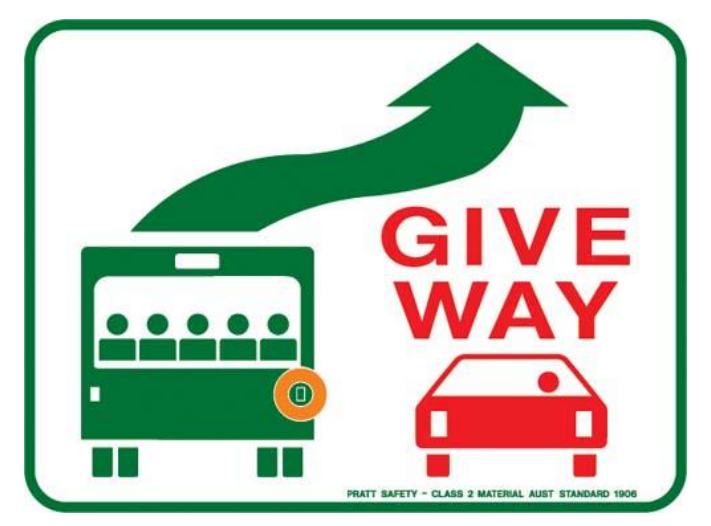

Figure 10: Give way to buses sign

Assume that a proportion of vehicles on the shoulder lane traffic, denoted by $\eta$, will give way to the leaving bus with an advisory sign "give way to bus". Then, the probability of an acceptance gap could be formulated as

$$
p=\left\{\begin{array}{lc}
1 ; & \text { if } \mathrm{T} \geq \tau^{*} \\
\eta ; & \text { otherwise }
\end{array}\right.
$$

where $T$ is the headway of the traffic in the shoulder lane; $\tau^{*}$ is the critical gap. Accordingly, the expected value of the probability is

$$
\bar{p}=P\left(T \geq \tau^{*}\right)+P\left(T<\tau^{*}\right) \times \eta
$$

The difference between probabilities calculated by eq. (12) and eq. (22) is the component of $P\left(T<\tau^{*}\right) \times \eta$. Assume $50 \%$ drivers will give way to buses, that is, $\eta=0.5$. For the impact analysis in Section 5.1 (traffic volume $=1080 \mathrm{veh} /$ hour and passengers' arrival rate $=3.3$ arrivals/min), the probability of more than one time doors opening and closing will be reduced to $6.68 \%$ from $30.1 \%$ according to eq. (14).

In reality, the impact of this sign should be analysed from the viewpoint of equity. The benefit of shoulder lane vehicles will be sacrificed if the sign is enforced by the transport agencies, although the bus dwell time will be significantly reduced. The sign will also affect lane changing, weaving, and merging activities. Therefore, it should be analysed in a much 
broader context by taking into account these activities in order to support the transport agencies.

\section{Conclusion and recommendation}

This study first pointed out that the bus dwell time at a bus bay possessed a high degree of uncertainty caused by the merging behaviour of buses to the traffic in the shoulder lane. A standard regenerative stochastic process is applied to formulate the interactions among buses, arriving passengers, and traffic on the shoulder lane and it enables us to estimate the probability distribution of the random dwell time at a bus bay. The case study and verification demonstrate the effectiveness of the proposed methodology to estimate the distribution of bus dwell time at a bus bay. The impact analysis of the advisory sign "give way to buses" would significantly decrease the dwell time and thus improve the bus capacity.

Following this study, two future works could be carried out. First, an econometric analysis could be conducted by taking into account the impact of give way to bus sign from the viewpoint of equity by taking into account the dwell time, lane changing, weaving, and merging activities. Second, a large scale application of the proposed model could be conducted to analyse the impact of bus bunching by incorporating the proposed model with off-the-shelf simulation software (e.g. Paramics).

\section{References}

Ceder, A., 2007. Public Transit Planning and Operation: Theory, Modeling and Practice. Butterworth-Heinemann, Oxford, UK.

Gu, W., Li, Y., Cassidy, M.J., and Griswold, J.B., 2011. On the capacity of isolated, curbside bus stops. Transportation Research Part B. 45: 714-723. 
Guenthner, R., and Hamat, K., 1988. Transit dwell time under complex fare structure. Journal of Transportation Engineering - ASCE. 114(3): 367-379.

Hadas, Y., and Ceder, A., 2010. Optimal coordination of public-transit vehicles using operational tactics examined by simulation. Transportation Research Part C. 19, 879895.

Jaiswal, S., Bunker, J., and Ferreira, L., 2010. Influence of platform walking on BRT station bus dwell time estimation: Australian analysis. Journal of Transportation Engineering - ASCE. 136(12): 1173-1179.

Kepaptsoglou, K. and Karlaftis, M., 2009. Transit Route Network Design Problem: Review. Journal of Transportation Engineering - ASCE, 135, 491-505.

Lam, W. H. K and Bell, M. G. H., 2003. Advanced Modeling for Transit Operations And Service Planning. Pergamon.

Levine, J., and Torng, G., 1994. Dwell time effects of low floor bus design. Journal of Transportation Engineering - ASCE. 120(6):914-929.

Levinson, H., 1983. Transit travel time performance. Transportation Research Record. 915 : $1-6$.

Lieu, H., N., Gartner, C.J., Messer, and A.K., Rathi, 1999. Traffic flow theory. U.S. Department of Transportation. Federal Highway Administration.

Lin, W.H., and R.L. Bertini, 2004. Modeling schedule recovery processes in transit operations for bus arrival time prediction. Journal of Advanced Transportation. 38, 347365.

Milkovits, M., 2008. Modeling the Factors Affecting Bus Stop Dwell Time: Use of Automatic Passenger Counting, Automatic Fare Counting, and Automatic Vehicle Location Data. Transportation Research Record: Journal of the Transportation Research Board. 2072, 125-130. 
Szeto, W.Y., Solayappan, M., and Jiang, Y., 2011a. Reliability-based transit assignment for congested stochastic transit networks. Computer-Aided Civil and Infrastructure Engineering, 26, 311-326.

Szeto, W.Y., Jiang, Y., Wong, K.I., and Solayappan, M., 2011b. Reliability-based stochastic transit assignment with capacity constraints: Formulation and solution method. Transportation Research Part C. http://dx.doi.org/10.1016/j.trc.2011.09.001

TCRP Report 19, 1996. Guidelines for the location and design of bus stops. Available at http:// gulliver.trb.org/publications/tcrp/tcrp_rpt_19-a.pdf Accessed on 16 January 2013.

Tirachini, A., 2011. Estimation of travel time and the benefits of upgrading the fare payment technology in urban bus services. Transportation Research Part $C$. doi:10.1016/j.trc.2011.11.007.

Tirachini, A., and Hensher, D.A., 2011. Bus congestion, optimal infrastructure investment and the choice of a fare collection system in dedicated bus corridors. Transportation Research Part B. 45: 828-844.

Tirachini, A., 2013. Bus dwell time: The effect of different fare collection systems, bus floor level and age of passengers. Transportmetrica A, 9, 28-49.

Yan, Y., Liu, Z., Meng, Q., and Jiang, Y., 2013. Robust optimization model of bus transit network design with stochastic travel time. Journal of Transportation Engineering ASCE. Published online ahead of print. doi: 10.1061/(ASCE)TE.1943-5436.0000536. 